\title{
AN IMPROVEMENT OF GRIBOV'S REGGEON CALCULUS
}

\author{
F.S. HENYEY and U.P. SUKHATME \\ Department of Physics, University of Michigan, Ann Arbor, Michigan 48104*
}

Received 24 June 1974

(Revised 20 September 1974)

\begin{abstract}
We derive an expression for Regge cuts and the associated enhancement of Regge poles, following Gribov's derivation of the reggeon calculus, but refraining from making an approximation made by Gribov. We show that Gribov's loop integrand should be multiplied by

$$
\left(\frac{\Delta\left(t, t_{1}, t_{2}\right)}{s_{\mathrm{th}} t}\right)^{j+1-\alpha_{1}-\alpha_{2}} \frac{\Gamma\left(\frac{1}{2}\right) \Gamma\left(j+2-\alpha_{1}-\alpha_{2}\right)}{\Gamma\left(j+\frac{3}{2}-\alpha_{1}-\alpha_{2}\right)} .
$$

This factor is identically unity for the Regge cut discontinuity, but is different from unity for enhanced singularities.
\end{abstract}

\section{Introduction}

Regge cuts are calculated from Feynman diagram models [1, 2], from $S$-matrix principles [3, 4] (involving $t$-channel unitarity), and from absorption models [5]. All these techniques, when handled properly, give identical expressions for the cut discontinuity, although phenomenological applications of the common expression have differed widely. A pictorial representation of a cut is shown in fig. 1 .

Accompanying the cut, there necessarily exists a phenomenon known as enhancement [3] of other singularities, which causes, for example, pole trajectories to be singular when they cross branch point trajectories. An example of such an enhancement is shown in fig. 2 . The cut also enhances itself, which causes the discontinuity to behave as $1 / \ln ^{2}\left(j-\alpha_{c}\right)$ near the branch point rather than the naively calculated constant. In ref. [3], the enhancement condition was solved by a $K$-matrix technique.

A more interesting technique for calculating cuts and solving the enhancement condition is the perturbation theory approach embodied in Gribov's reggeon calculus [2]. This theory has the structure of a field theory in which there are two space dimensions and the angular momentum plays the role of energy. This feature allows powerful field theory techniques to be applied, notably renormalization group tech-

\footnotetext{
${ }^{*}$ Research supported by the US Atomic Energy Commission.
} 


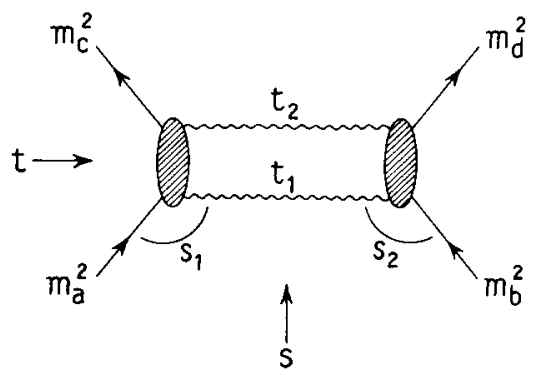

Fig. 1. The general diagram for calculating regge cuts, and associated enhancements. The kinematic variables are shown. In general, the two "vertices" have high energy $j$-plane singularities, for which this diagram is an enhancement.

niques [6]. When such techniques are applied, it might be very important that the theory be exactly correct. In this paper, we demonstrate that Gribov's expression is not exactly correct, and we derive the correct expression.

The difficulty with the reggeon calculus was first noticed by Hayot and Sukhatme [7] in a phenomenological application of the reggeon calculus. (In particular, Gribov's technique does not give the correct threshold factor for the two-pion cut.)

The source of the difficulty is a standard high-energy approximation that Gribov made in his derivation. It can be verified that this approximation is valid for the calculation of the cut discontinuity. (Most easily by comparing the results of refs. [2, 3]). It turns out, however, that the approximation is invalid for the enhancement. The approximation need not be made, and the derivation can still be completed by use of techniques now available. In the appendix we carry out the derivation, using a technique taken from a paper (on the diagonalization of Bethe-Salpeter equations) by Abarbanel and Saunders [8].

The result found differs from Gribov's result simply by a factor in the integrand. In the following sections we present the result and discuss the implications.

\section{The improved reggeon calculus}

We assume that the reader is familiar with Gribov' rules [2] as we present the improvement. We are interested in calculating a loop shown in fig. 1. Details are given in the appendix. In eq. (A.1), because of the presence of uncalculated daughter tra-

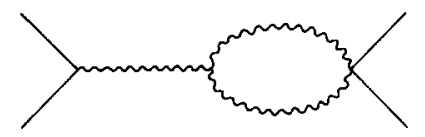

Fig. 2. A reggeon calculus diagram exhibiting an enhancement. Gribov's approximation is invalid for the enhancement, that is, for the Regge pole residue of this diagram. 
jectories, a choice must be made for the continuation of the reggeon exchange to large values of the external masses. We choose the form $\left(s / M_{1}^{2} M_{2}^{2}\right)^{\alpha}$, assigning terms proportional to $(1 / t)\left(s / M_{1}^{2} M_{2}^{2}\right)^{\alpha-1}$, etc., to be included with the daughter trajectories.

In what follows, we use the $t$-channel orbital angular momentum variable [3]

$$
l=j-\alpha_{1}\left(t_{1}\right)-\alpha_{2}\left(t_{2}\right),
$$

where $j$ is the $t$-channel angular momentum and $\alpha_{1}\left(t_{1}\right)$ and $\alpha_{2}\left(t_{2}\right)$ are the exchanged Regge poles. The Gribov vertices are given in terms of the 2-particle - 2-reggeon amplitude by

$$
\begin{aligned}
& N_{\mathrm{L}, \mathrm{R}}\left(j, t ; t_{1}, t_{2}\right)=\int \mathrm{d} s^{\prime}\left(s^{\prime}\right)^{-\alpha_{1}-\alpha_{2}} \\
& \quad \times \frac{\left(P_{\mathrm{L}, \mathrm{R}} P_{\mathrm{I}}\right)^{-l-1} \Gamma\left(l+\frac{3}{2}\right)}{\sqrt{\pi} \Gamma(l+1)} Q_{l}\left(y^{\prime}\right) \operatorname{Im} M_{\mathrm{L}, \mathrm{R}}\left(s^{\prime}, t ; t_{1}, t_{2}\right),
\end{aligned}
$$

where the variables $y^{\prime}, P_{\mathrm{L}, \mathrm{R}}, P_{\mathrm{I}}$ are defined in the appendix. The variable of importance is

$$
P_{\mathrm{I}}=\left(\frac{\Delta\left(t, t_{1}, t_{2}\right)}{4 t}\right)^{\frac{1}{2}},
$$

and where $\Delta$ is the usual triangle function given in eq. (7).

The exact choice of the factors in eq. (2) makes $N$ have the same residues of $j$ plane singularities as Gribov's expression

$$
N^{\prime}=\int_{S_{\text {th }}}^{\infty} \mathrm{d} s^{\prime} s^{\prime-l-1} \operatorname{Im} M .
$$

The $j$-plane amplitude is then given by the expression

$$
\begin{gathered}
M(j, t)=\int_{\Delta<0} \frac{\mathrm{d} t_{1} \mathrm{~d} t_{1}}{\left(-\Delta\left(t, t_{1}, t_{2}\right)\right)^{\frac{1}{2}}} s_{\mathrm{th}}^{l+1} P\left(\alpha_{1}\left(t_{1}\right)\right) P\left(\alpha_{2}\left(t_{2}\right)\right) \\
\times \frac{1}{l+1} N_{\mathrm{L}}\left(j, t ; t_{1}, t_{2}\right) N_{\mathrm{R}}\left(j, t ; t_{1}, t_{2}\right) F\left(j, t ; t_{1}, t_{2}\right),
\end{gathered}
$$

where $P$ is the reggeon propagator usually expressed as

$$
\begin{aligned}
& P(\alpha)=\frac{1}{\sin \pi \alpha} \times \text { signature factor, } \\
& \Delta\left(t, t_{1}, t_{2}\right)=t^{2}+t_{1}^{2}+t_{2}^{2}-2 t t_{1}-2 t t_{2}-2 t_{1} t_{2},
\end{aligned}
$$


and the only difference with Gribov is the presence of the factor:

$$
F\left(j, t ; t_{1}, t_{2}\right)=\left(\frac{\Delta\left(t, t_{1}, t_{2}\right)}{t s_{\text {th }}}\right)^{l+1} \frac{\sqrt{\pi} \Gamma(l+2)}{\Gamma\left(l+\frac{3}{2}\right)}
$$

$s_{\text {th }}$ is the minimum value of $s_{1}, s_{2}$, and $s$ in Gribov's Mellin transform (see eq. (4)). The exact expression (eq. (5)) is independent of $s_{\text {th }}$, although of course Gribov's expression obtained by setting $F=1$ is not.

Finally, the high-energy behavior of the diagram is given by

$$
M(s, t)=\frac{1}{2 \pi i} \int_{c-i \infty}^{c+i \infty} s^{j} M(j, t) \mathrm{d} j .
$$

The expressions presented here are derived in the appendix.

In addition to the factor $F$, we differ from Gribov in requiring the FroissartGribov definition of $N$ (eq. (2)) instead of the Mellin transform definition (eq. (4)). In eq. (5), we have not retained factors of 2 and $\pi$ properly, since these can be recovered if desired by requiring that our results would agree with Gribov's if we were to set $F=1$. Of course, both Gribov and ourselves always assume $s$ is large; we do not assume $s_{1}$ and $s_{2}$ are small.

\section{Implications}

In sect. 2, the main result presented is the presence of the factor $F$ given by eq. (8). Gribov's approximation amounts to

$$
F \approx 1 \quad \text { (Gribov). }
$$

Thus the sole modification of the reggeon calculus is to introduce an $F$ factor for each loop. The leading Regge cut (and some other singularities) comes from a pinch involving the pole of $1 /(l+1)$ at $l=-1$. From eq. (8), one immediately sees that

$$
(F)_{l=-1} \equiv 1 \text {, }
$$

which confirms that Gribov calculated the cut discontinuity correctly. Gribov's Mellin transform and our Froissart-Gribov transform also become equal at $l=-1$. Eq. (8) has other interesting properties. $F$ is a function only of the variables $j, t, t_{1}$ and $t_{2}$, but is independent of, for example, the external masses. Therefore, this factor can be associated completely with the loop, and our result immediately generalizes to multiloop diagrams such as that shown in fig. 3 .

Daughter cuts are explicit from the factor $\Gamma(l+2)$. (Unfortunately, however, the leading cuts from the uncalculable daughter poles are as important as the daughter cuts.) It is readily verified that the $t$-channel threshold behavior is correctly given by the inclusion of the $F$ factor; although incorrectly given without that factor. 


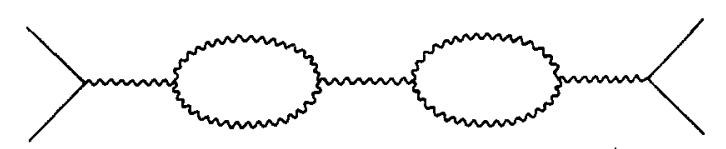

Fig. 3. A two-loop diagram for which our result readily generalizes. The appropriate factor $F$ is associated with each loop.

The expression for $F$ becomes singular at the triple-pomeron (3P) point $t=t_{1}=$ $=t_{2}=0, \alpha(t)=1+\alpha^{\prime} t$.

At this point, we find

$$
F \approx\left(\frac{\Delta}{t s_{\text {th }}}\right)^{l+1}=\left(\frac{t_{1}+t_{2}-t-\left(t_{1}-t_{2}\right)^{2} / t}{s_{\text {th }}}\right)^{l+1}
$$

The $t_{1}-t_{2}$ integration causes no problem so

$$
F \approx\left(\frac{t_{1}+t_{2}-t}{s_{\text {th }}}\right)^{l+1},
$$

if we approach the 3P point along the cut $l=-1$ and $F=1$. If on the other hand we approach along the pole $j=\alpha$

$$
l+1=j-\alpha_{1}-\alpha_{2}+1=\alpha-\alpha_{1}-\alpha_{2}+1=\alpha^{\prime}\left(t-t_{1}-t_{2}\right)
$$

So

$$
F \approx\left(\frac{t_{1}+t_{2}-t}{s_{\text {th }}}\right)^{\alpha^{\prime}\left(t-t_{1}-t_{2}\right)},
$$

which does, in fact, approach 1, but with a singularity. The applications [6] assuming $F=1$ are thus probably reliable, but should be checked.

Near $t=0$, the expression for $F$ (eq. (8)) can be simplified. As $t=0$ is approached, $t_{1}$ and $t_{2}$ become nearly the same. As a result, an integral over $t_{1}-t_{2}$ can be explicitly evaluated. We let $\sigma=t_{1}+t_{2}$ and $\epsilon=t_{1}-t_{2}$.

Our integral is (eq. (5))

$$
\begin{aligned}
M & =\int F s_{\text {th }}^{l+1} \frac{N_{\mathrm{L}} N_{\mathrm{R}} P\left(t_{1}\right) P\left(t_{2}\right)}{l+1} \frac{\mathrm{d} \sigma \mathrm{d} \epsilon}{\left(-t^{2}+2 t \sigma-\epsilon^{2}\right)^{\frac{1}{2}}} \\
& =\int \frac{N_{\mathrm{L}} N_{\mathrm{R}} P\left(t_{1}\right) P\left(t_{2}\right)}{l+1} \frac{\sqrt{\pi} \Gamma(l+2)}{\Gamma\left(l+\frac{3}{2}\right)} \frac{\mathrm{d} \sigma \mathrm{d} \epsilon}{t^{\frac{1}{2}}}\left(2 \sigma-t-\epsilon^{2} / t\right)^{l+\frac{1}{2}} .
\end{aligned}
$$

The $\epsilon$ integral can be done because at $t=0$ the $N^{\prime} s, P^{\prime}$ s and $l$ become independent of $\epsilon$. 
We find

$$
M=\pi \int \frac{N_{\mathrm{L}} N_{\mathrm{R}} P_{1} P_{2}}{l+1} \mathrm{~d} \sigma(2 \sigma-t)^{l+1}
$$

in place of Gribov's

$$
M_{G}=\pi \int \frac{N_{\mathrm{L}} N_{\mathrm{R}} P_{1} P_{2}}{l+1} \mathrm{~d} \sigma s_{\mathrm{th}}^{l+1} .
$$

Therefore, at $t=0, F$ can be replaced by

$$
F^{\prime}=\left(\frac{2 \sigma}{s_{\text {th }}}\right)^{l+1}
$$

Notice that the gamma functions in $F$ have been cancelled at $t=0$ in doing the $t_{1}-t_{2}$ integration.

As a simple example of this improvement of Gribov's formula, we can investigate the enhancement of an arbitrary (vacuum quantum number) trajectory by the two pomeron cut. As an approximation, we consider the two pomeron cut concentrated at its branch point, $\alpha_{1}=\alpha_{2}=1$. Then we find

$$
l=\alpha_{0}-\alpha_{1}-\alpha_{2}=\alpha_{0}-2 \text {. }
$$

Assuming that $s_{\mathrm{th}}$ is $1 \mathrm{GeV}^{2}$ and assuming, for definiteness

$$
s_{\text {th }}^{l+1} \frac{N_{\mathrm{L}} N_{\mathrm{R}} P_{1} P_{2}}{l+1}=\mathrm{e}^{5 \sigma} \quad(\sigma \text { negative) },
$$

evaluation of Gribov's expression (eq. (18)) gives

$$
M_{\mathrm{G}}=\pi \int_{-\infty}^{0} \mathrm{e}^{5 \sigma} \mathrm{d} \sigma=\frac{1}{5} \pi,
$$

while the improved expression (17) gives

$$
M=\pi \int_{-\infty}^{0} \mathrm{e}^{5 \sigma}(2 \sigma)^{\alpha_{0}-1} \mathrm{~d} \sigma=\pi 2^{\alpha_{0}-1} \Gamma\left(\alpha_{0}\right) 5^{-\alpha_{0}} .
$$

If we consider the $\mathrm{P}^{\prime}$ trajectory with $\alpha_{0}=\frac{1}{2}$, we get

$$
M=\pi\left(\frac{1}{10} \pi\right)^{\frac{1}{2}},
$$

which is a factor of 2.8 larger than Gribov's expression gives.

Of course, the detailed numerical assumptions are not to be trusted, but this little example shows that the correction factor can be quantitatively important. 


\section{Conclusion}

We have demonstrated that Gribov's reggeon calculus is improved by multiplying each loop integral by an appropriate factor. This factor arises from treating the kinematics completely rather than making an approximation which is not always valid. Our factor is identically unity for the cut discontinuity, but can be quantitatively and even qualitatively important in a number of places, for example in calculating the enhancement of Regge poles.

We wish to thank $\mathrm{H}$. Abarbanel for kindly informing us about the technique of ref. [8] which allowed us to do this calculation. We gratefully acknowledge many fruitful discussions and helpful suggestions from F. Hayot and G. Kane.

\section{Appendix}

Our starting expression for the two-reggeon cut is

$$
\begin{aligned}
& M(s, t)=\int \mathrm{d}^{4} P^{\prime} A_{\mathrm{L}}\left(s_{1}, t, \alpha_{1}, \alpha_{2}, m_{\mathrm{a}}^{2}, m_{\mathrm{c}}^{2}\right), \\
& A_{\mathrm{R}}\left(s_{2}, t, \alpha_{1}, \alpha_{2}, m_{\mathrm{b}}^{2}, m_{\mathrm{d}}^{2}\right) P\left(t_{1}\right) P\left(t_{2}\right)\left(\frac{s}{s_{1} s_{2}}\right)^{\alpha_{1}+\alpha_{2}}
\end{aligned}
$$

where $A_{\mathrm{L}}$ and $A_{\mathrm{R}}$ correspond to the absorptive parts of the particle reggeon amplitudes, $\alpha_{1,2} \equiv \alpha_{1}\left(t_{1}\right), \alpha_{2}\left(t_{2}\right)$ and the kinematic variables are defined in fig. 1 . If $s_{1}$ and/or $s_{2}$ are large, $A_{\mathrm{L}}$ and/or $A_{\mathrm{R}}$ would contain Regge poles, etc., and an enhanced graph would result. It could be argued that the factors $s^{\alpha} i(i=1,2)$ appearing in eq. (A.1) should actually be $P_{\alpha_{i}}\left(-z_{t_{i}}\right)$, where $z_{t_{i}}$ is linear in $s$. The two choices agree at $s \gg s_{1} s_{2}$. However, good analyticity properties at $t_{i}=0$ necessarily require the presence of an infinite sequence of Regge daughters lying below the parent, located such that the full family is well behaved [9]. The expression for the reggeons in eq. (A.1) is therefore necessarily somewhat ambiguous. Our choice has the correct leading asymptotic behavior and good analyticity properties.

We consider the remainder of the $P_{\alpha}$ to be associated with the daughters, and its contributions to be included with the daughters. These contributions will, of course, also enhance the singularities of $A_{\mathrm{L}}$ and $A_{\mathrm{R}}$. In order to correctly determine exactly the enhancement caused by a particular hybrid Feynman diagram, one would have to calculate the ladder exchange amplitude exactly, not only its high energy limit. Needless to say, this is beyond present day capabilities. However, in keeping with the spirit of the reggeon calculus, we may hope that the enhancement of high-lying singularities caused by daughters, etc., will be smaller than the contribution we calculate.

Furthermore, in any case, we shall see that the factor $F$ which we obtain as an 
improvement of the reggeon calculus stems from a different origin. It basically comes from a careful treatment of the Jacobian in the transformation of variables $\mathrm{d}^{4} P^{\prime}=J \mathrm{~d} s_{1} \mathrm{~d} s_{2} \mathrm{~d} t_{1} \mathrm{~d} t_{2}$, which is discussed and used in an approximate way by Gribov [2]. Therefore our final result for the modifying factor $F$ does not depend crucially on the precise form for the integrand chosen in eq. (A.1). It is convenient to introduce the following quantities:

$$
P_{\mathrm{L}} \equiv \sqrt{\frac{\Delta\left(t, m_{\mathrm{a}}^{2}, m_{\mathrm{c}}^{2}\right)}{4 t}}, \quad P_{\mathrm{R}} \equiv \sqrt{\frac{\Delta\left(t, m_{\mathrm{b}}^{2}, m_{\mathrm{d}}^{2}\right)}{4 t}}, \quad P_{\mathrm{I}} \equiv \sqrt{\frac{\Delta\left(t, t_{1}, t_{2}\right)}{4 t}} .
$$

Defining the partial-wave amplitude $M(j, t)$ by

$$
M(j, t) \equiv \int_{s_{\mathrm{th}}}^{\infty} \mathrm{d} s \Phi M(s, t) Q_{j}(y), \quad \Phi \equiv\left[\frac{\left(P_{\mathrm{L}} P_{\mathrm{R}}\right)^{-j-1} \Gamma\left(j+\frac{3}{2}\right)}{\sqrt{\pi} \Gamma(j+1)}\right],
$$

where the factor $\Phi$ is chosen such that $M(j, t)$ is the usual Mellin transform for large $s$ and $y=y(s, t)$ is given below, we obtain

$$
\begin{aligned}
& M(j, t)=\int \mathrm{d}^{4} P^{\prime} \int_{s_{\mathrm{th}}}^{\infty} \mathrm{d} s\left\{\frac{A_{\mathrm{L}} A_{\mathrm{R}} P\left(t_{1}\right) P\left(t_{2}\right)}{\left(s_{1} s_{2}\right)^{\alpha_{1}+\alpha_{2}}}\right\} s^{\alpha_{1}+\alpha_{2}} Q_{j}(y), \\
& \quad \times\left[\frac{\left(P_{\mathrm{L}} P_{\mathrm{R}}\right)^{-j-1} \Gamma\left(j+\frac{3}{2}\right)}{\sqrt{\pi} \Gamma(j+1)}\right] .
\end{aligned}
$$

The next step in deriving the reggeon calculus rules consists of changing integration variables from the loop four-momentum $P^{\prime}$ to $\left(t_{1}, t_{2}, s_{1}, s_{2}\right)$.

$$
\begin{aligned}
& M(j, t)=\int \frac{\mathrm{d} t_{1} \mathrm{~d} t_{2} \mathrm{~d} s_{1} \mathrm{~d} s_{2} \theta(-D)}{\sqrt{-D}} \int_{s_{\text {th }}}^{\infty} \mathrm{d} s\left\{\frac{A_{\mathrm{L}} A_{\mathrm{R}} P\left(t_{1}\right) P\left(t_{2}\right)}{\left(s_{1} s_{2}\right)^{\alpha_{1}+\alpha_{2}}}\right\} s^{\alpha_{1}+\alpha_{2}} \\
& \quad \times\left[\frac{\left(P_{\mathrm{L}} P_{\mathrm{R}}\right)^{-j-1} \Gamma\left(j+\frac{3}{2}\right)}{\sqrt{\pi} \Gamma(j+1)}\right] Q_{j}(y),
\end{aligned}
$$

where

$$
\frac{\theta(-D)}{\sqrt{-D}}=\frac{\partial\left(P_{0}^{\prime}, P_{1}^{\prime}, P_{2}^{\prime}, P_{3}^{\prime}\right)}{\partial\left(t_{1}, t_{2}, s_{1}, s_{2}\right)}
$$

At this stage, Gribov approximated $D$ by

$$
D_{\text {approx }}=s^{2} \Delta\left(t, t_{1}, t_{2}\right),
$$


and performed the $s, s_{1}$ and $s_{2}$ integrations. Such an approximation is not necessary and as pointed out in the text, it leads to difficulties. Here we show that the integrations over $s_{1}, s_{2}, s$ can in fact in accomplished without approximation by means of another change of variables, the group-theoretical motivation for which is discussed in detail by Abarbanel and Saunders [8]. The new integration variables $\left(t_{1}, t_{2}, y_{1}\right.$, $\left.y_{2}, y\right)$ are given by

$$
y=\frac{s}{2 P_{\mathrm{L}} P_{\mathrm{R}}}-\sigma, \quad y_{1}=\frac{s_{1}}{2 P_{\mathrm{I}} P_{\mathrm{I}}}-\sigma_{1}, \quad y_{2}=\frac{s_{2}}{2 P_{\mathrm{I}} P_{\mathrm{R}}}-\sigma_{2},
$$

where $\sigma, \sigma_{1}, \sigma_{2}$ are independent of $s, s_{1}, s_{2}$. (The interested reader can readily ob. tain explicit expressions for $\sigma, \sigma_{1}, \sigma_{2}$, which do not concern us in this paper, by studying ref. [8].)

The expression for the cut now becomes

$$
\begin{aligned}
& M(j, t)=\int \frac{P_{\mathrm{L}} P_{\mathrm{R}} P_{\mathrm{I}} \mathrm{d} t_{1} \mathrm{~d} t_{2} \mathrm{~d} y_{1} \mathrm{~d} y_{2} \mathrm{~d} y Q_{j}(y) \theta\left(y^{2}+y_{1}^{2}+y_{2}^{2}-1-2 y y_{1} y_{2}\right)}{\sqrt{t}\left(y^{2}+y_{1}^{2}+y_{2}^{2}-1-2 y y_{1} y_{2}\right)^{\frac{1}{2}}} \\
& \quad \times\left\{\frac{A_{\mathrm{L}} A_{\mathrm{R}} P\left(t_{1}\right) P\left(t_{2}\right)}{\left(s_{1} s_{2}\right)^{\alpha_{1}+\alpha_{2}}}\right\} s^{\alpha_{1}+\alpha_{2}}\left[\frac{\left(P_{\mathrm{L}} P_{\mathrm{R}}\right)^{-j-1} \Gamma\left(j+\frac{3}{2}\right)}{\sqrt{\pi} \Gamma(j+1)}\right] .
\end{aligned}
$$

For large values of $s$,

$$
\begin{aligned}
& Q_{j}(y) s^{\alpha_{1}+\alpha_{2}}\left[\frac{\left(P_{\mathrm{L}} P_{\mathrm{R}}\right)^{-j-1} \Gamma\left(j+\frac{3}{2}\right)}{\sqrt{\pi} \Gamma(j+1)}\right]=s^{-j-1+\alpha_{1}+\alpha_{2}} \\
& \quad=Q_{l}(y)\left[\frac{\left(P_{\mathrm{L}} P_{\mathrm{R}}\right)^{-l-1} \Gamma\left(l+\frac{3}{2}\right)}{\sqrt{\pi} \Gamma(l+1)}\right]
\end{aligned}
$$

where we have defined the quantity $l \equiv j-\alpha_{1}-\alpha_{2}$, which can be interpreted as "orbital" angular momentum [3]. Let us make this change in the expression for $M(j, t)$

$$
\begin{aligned}
& M(j, t)=\int \frac{P_{\mathrm{L}} P_{\mathrm{R}} P_{\mathrm{I}} \mathrm{d} t_{1} \mathrm{~d} t_{2} \mathrm{~d} y_{1} \mathrm{~d} y_{2} \mathrm{~d} y Q_{l}(y) \theta\left(y^{2}+y_{1}^{2}+y_{2}^{2}-1-2 y y_{1} y_{2}\right)}{\sqrt{t}\left(y^{2}+y_{1}^{2}+y_{2}^{2}-1-2 y y_{1} y_{2}\right)^{\frac{1}{2}}} \\
& \quad \times\left\{\frac{A_{\mathrm{L}} A_{\mathrm{R}} P\left(t_{1}\right) P\left(t_{2}\right)}{\left(s_{1} s_{2}\right)^{\alpha_{1}+\alpha_{2}}}\right\}\left[\frac{\left(P_{\mathrm{L}} P_{\mathrm{R}}\right)^{-l-1} \Gamma\left(l+\frac{3}{2}\right)}{\sqrt{\pi} \Gamma(l+1)}\right] .
\end{aligned}
$$

The $y$ integration can be carried out by means of the following result [8]: 


$$
\int_{1}^{\infty} \mathrm{d} y Q_{l}(y) \frac{\theta\left(y^{2}+y_{1}^{2}+y_{2}^{2}-1-2 y y_{1} y_{2}\right)}{\left(y^{2}+y_{1}^{2}+y_{2}^{2}-1-2 y y_{1} y_{2}\right)^{\frac{1}{2}}}=Q_{l}\left(y_{1}\right) Q_{l}\left(y_{2}\right)
$$

This remarkable integral separates the $y_{1}, y_{2}$ dependence of the integrand into a factorized form.

$$
\begin{aligned}
& M(j, t)=\int \frac{P_{\mathrm{L}} P_{\mathrm{R}} P_{\mathrm{I}} \mathrm{d} t_{1} \mathrm{~d} t_{2} \mathrm{~d} y_{1} \mathrm{~d} y_{2}}{\sqrt{t}}\left\{\frac{A_{\mathrm{L}} A_{\mathrm{R}} P\left(t_{1}\right) P\left(t_{2}\right)}{\left(s_{1} s_{2}\right)^{\alpha_{1}+\alpha_{2}}}\right\}\left[\frac{\left(P_{\mathrm{L}} P_{\mathrm{R}}\right)^{-l-1} \Gamma\left(l+\frac{3}{2}\right)}{\sqrt{\pi} \Gamma(l+1)}\right] \\
& \quad \times Q_{l}\left(y_{1}\right) Q_{l}\left(y_{2}\right) .
\end{aligned}
$$

The partial wave particle-Reggeon amplitudes are defined by

$$
N_{\mathrm{L}, \mathbf{R}}(j, t)=\int \mathrm{d} y_{1,2} 2 P_{\mathrm{L}, \mathbf{R}} P_{\mathrm{I}} A_{\mathrm{L}, \mathrm{R}} s_{1,2}{ }^{-\alpha_{1}-\alpha_{2}} Q_{l}\left(y_{1,2}\right)\left[\frac{\left(P_{\mathrm{L}, \mathrm{R}} P_{\mathrm{I}}\right)^{-l-1} \Gamma\left(l+\frac{3}{2}\right)}{\sqrt{\pi} \Gamma(l+1)}\right],
$$

where the multiplicative factor has again been chosen such that one obtains a Mellin transform for large $s_{1,2}$. The final result is

$$
M(j, t)=\int \frac{\sqrt{\pi} \Gamma(l+2)\left(P_{\mathrm{I}}\right)^{2 l+2} \mathrm{~d} t_{1} \mathrm{~d} t_{2} P\left(t_{1}\right) P\left(t_{2}\right) N_{\mathrm{L}} N_{\mathrm{R}}}{(l+1) \Gamma\left(l+\frac{3}{2}\right) 4 P_{\mathrm{I}} \sqrt{t}} .
$$

The expression corresponding to eq. (A.15) in Gribov's approach is obtained by taking eqs. (A.9) and (A.10) and setting

$$
\sigma, \sigma_{1}, \sigma_{2} \rightarrow 0, \quad\left(y^{2}+y_{1}^{2}+y_{2}^{2}-1-2 y y_{1} y_{2}\right)^{\frac{1}{2}} \rightarrow y .
$$

The limits of integration are $s_{\text {th }} \leq s_{1}, s_{2} \leq s$.

$$
M_{\mathrm{G}}(j, t)=\int \frac{P_{\mathrm{L}} P_{\mathrm{R}} P_{\mathrm{I}}}{\sqrt{t}} \mathrm{~d} t_{1} \mathrm{~d} t_{2} \mathrm{~d} y_{1} \mathrm{~d} y_{2} \mathrm{~d} y \frac{s^{-l-1}}{y}\left\{\frac{A_{\mathrm{L}} A_{\mathrm{R}} P\left(t_{1}\right) P\left(t_{2}\right)}{\left(s_{1} s_{2}\right)^{\alpha_{1}+a_{2}}}\right\}
$$

with

$$
y=\frac{s}{2 P_{\mathrm{L}} P_{\mathrm{R}}}, \quad y_{1}=\frac{s_{1}}{2 P_{\mathrm{L}} P_{\mathrm{I}}}, \quad y_{2}=\frac{s_{2}}{2 P_{\mathrm{R}} P_{\mathrm{I}}} .
$$

Gribov's definition of partial wave amplitudes is

$$
A_{\mathrm{L}, \mathrm{R}}=\int N_{\mathrm{L}, \mathrm{R}}\left(j_{1,2}\right) s_{1,2}^{j_{1,2}} \mathrm{~d} j_{1,2}
$$

Substitution of eq. (A.17) in eq. (A.16) allows the $y_{1}, y_{2}, y$ integrations to be performed. At this stage the result is 


$$
\begin{aligned}
& M_{\mathrm{G}}(j, t)=\int s_{\mathrm{th}}-l+1+l_{1}+l_{2} \mathrm{~d} l_{1} \mathrm{~d} l_{2} \frac{\mathrm{d} t_{1} \mathrm{~d} t_{2} N_{\mathrm{L}}\left(l_{1}\right) N_{\mathrm{R}}\left(l_{2}\right)}{P_{\mathrm{I}} \sqrt{t}\left(l_{1}+1\right)\left(l_{2}+1\right)} \\
& \quad \times\left[\frac{1}{l-l_{1}-l_{2}-1}-\frac{1}{l-l_{1}}-\frac{1}{l-l_{2}}+\frac{1}{l+1}\right] .
\end{aligned}
$$

Gribov's final result is obtained by doing the $l_{1}, l_{2}$ integrals.

$$
M_{\mathrm{G}}(j, t)=\int \frac{\mathrm{d} t_{1} \mathrm{~d} t_{2} P\left(t_{1}\right) P\left(t_{2}\right) N_{\mathrm{L}} N_{\mathrm{R}}}{4 P_{\mathrm{I}} \sqrt{t}(l+1)} s_{\mathrm{th}}{ }^{l+1}
$$

A comparison of the above expression with the correct result eq. (A.15) shows that the additional factor $F$ is given by

$$
F=\frac{\sqrt{\pi} \Gamma(l+2)\left(P_{\mathrm{I}}\right)^{2 l+2}}{\Gamma\left(l+\frac{3}{2}\right) s_{\mathrm{th}}^{l+1}}=\left[\frac{\Delta\left(t, t_{1}, t_{2}\right)}{4 t s_{\mathrm{th}}}\right]^{l+1} \frac{\sqrt{\pi} \Gamma(l+2)}{\Gamma\left(l+\frac{3}{2}\right)} .
$$

\section{References}

[1] S. Mandelstam, Nuovo Cimento 30 (1963) 1113, $1127,1143$.

[2] V.N. Gribov, JETP (Sov. Phys.) 26 (1968) 414.

[3] V.N. Gribov, I. Ya Pomeranchuk and K.A. Ter-Martirosyan, Yad. Fiz. 2 (1965) 361; Sov. J. Nucl. Phys. 2 (1966) 258.

[4] A.R. White, Nucl. Phys. B39 (1972) 432, 461.

[5] F. Henyey, G.L. Kane, J. Pumplin and M.H. Ross, Phys. Rev. 182 (1969) 1579; M.H. Ross, F.S. Henyey, G.L. Kane, Nucl. Phys. B23 (1970) 269.

[6] H.D.I. A barbanel and J.B. Bronzan, NAL preprint (1973); A.A. Migdal, A.M. Polyakov and K.A. Ter-Martirosyan, Phys. Letters B48 (1974) 239; Preprint ITEP-102.

[7] F. Hayot and U.P. Sukhatme, private communication.

[8] H.D.I. Abarbanel and L.M. Saunders, Phys. Rev. D2 (1970) 711.

[9] D.Z. Freedman and J.M. Wang, Phys. Rev. 153 (1967) 1596; M. Goldberger and C.E. Jones, Phys. Rev. 150 (1966) 1269. 